\title{
RADIATIVE TRANSFER MODELING OF MICROWAVE EMISSION AND DEPENDENCE ON FIRN PROPERTIES
}

\author{
J. C. Comiso and H. J. Zwally \\ (Goddard Laboratory for Atmospheric Sciences, NASA/Goddard Space Flight Center, Greenbelt, \\ Maryland 20771, U.S.A.)
}

and J. L. Saba

(Computer Sciences Corporation, Silver Spring, Maryland 20910, U.S.A.)

\section{ABSTRACT}

The microwave emission from a model polar firn was calculated using a numerical solution of the radiative transfer equation that included angledependent Rayleigh scattering. The depth-dependent parameters in the equation were physical temperature and the coefficients of scattering and absorption. The coefficients were based on Rayleigh scattering from the snow grains. The bulk emissivity and the seasonal dependence of brightness temperature were calculated for seven locations at which grain sizes were measured as a function of depth. When the absorption and scattering coefficients are adjusted, the modeled emissivities agree with observed emissivities at these locations. The modeled seasonal dependence of brightness temperatures also compares well with values obtained at $1.55 \mathrm{~cm}$ wavelength by the Nimbus -5 satellite. Good agreement with data did not occur when the imaginary part of the index of refraction (and, hence, the absorption coefficient) had a significant temperature dependence between 210 and $250 \mathrm{~K}$.

\section{INTRODUCTION}

The unexpectedly low microwave brightness temperatures of the Greenland and Antarctic ice sheets, as measured by the electrically scanning microwave radiometer (ESMR) on Nimbus-5, have been the subject of several theoretical investigations in recent years. Chang and others (1976) showed that, for a uniform snow medium, volume scattering is a dominant factor affecting the microwave emission and that the source of radiation can emanate from several meters within the medium. Subsequently, taking into account variations of snow properties with depth, Zwally (1977) used an analytic approximation to solve the microwave emissivity of the polar firn. The approximation overestimated the effect of radiative scattering in the medium because contributions due to multiple scattering were neglected. The scattering coefficient $\gamma_{s}$ had to be multiplied by an empirical parameter $f=0.12$ to obtain agreement between calculated emissivities, $\varepsilon$ (model) and observed emissivities $\varepsilon$ (obs). Using a different numerical technique for solving the radiative transfer equation from the one used in this paper, Chang and others (1980) showed good quantitiative agreement with their quoted bright- ness temperatures from Nimbus-5, but the quoted values differ substantially from other published values (Zwally 1977, Zwally and Gloersen 1977). This paper presents the result of a numerical solution of the radiative transfer equation similar to the method of Chang and others (1976), but with depth-dependent parameters. When a value of $f=0.3$ is used to adjust the scattering coefficient, the results agree with observed microwave emissivities and qualitatively with Zwally's results. In addition, the model is used to simulate the seasonal variation of the microwave emission. Comparison of modeled and observed seasonal amplitudes provides additional information on the validity of the radiative transfer model and, in particular, of the values of the scattering and absorption parameters used in the model.

\section{RADIATIVE TRANSFER MODEL}

The intensity (energy flux per unit wavelength per unit solid angle) of radiation $I(\theta)$ passing through a medium at an angle $\theta$ with the vertical can be calculated from the equation of radiative transfer given by:

$$
\begin{aligned}
& I(\theta)=I(\tau, \theta) e^{-\tau / \mu}+\int_{\tau}^{0}\left\{\varepsilon\left(\tau^{\prime}\right) B\left[T\left(\tau^{\prime}\right)\right] \frac{\left.e^{-\tau j \mu}\right\} d \tau^{\prime}+}{\mu}\right. \\
& \left.+\frac{1}{2} \int_{\tau}^{0}\left\{\int_{0}^{\pi} P\left(\theta^{\prime}\right) I\left(\tau^{\prime}, \theta^{\prime}\right) \sin \theta^{\prime} d \theta^{\prime}\right\} \omega_{0}\left(\tau^{\prime}\right) \frac{e^{-\tau j}}{\mu}{ }^{\mu}\right\} d \tau^{\prime},
\end{aligned}
$$

where $t$ is the optical depth of the medium, $\varepsilon$ is the emittance, $\omega_{0}$ is the single scattering albedo, $B$ is the Rayleigh-Jeans approximation to the Planck function, $\mu=\cos \theta, P(\theta)$ is the scattering phase function, and $\tau^{\prime}$ and $\theta^{\prime}$ are the integration variables for optical depth and scattering angle respectively (cf. Swihart 1971). The first term on the right-hand side of the equation is the contribution of radiation incident on the mediun from the botton and attenuated by the medium before it reaches the surface. The second term, the non-scattering term, takes into account enission and absorption within the medium, whereas the third term is the contribution to the radiation due to scattering. The brightness temperature $T_{B}$ is related to intensity by: 


$$
T_{B}=\frac{\lambda^{4} I}{2 c k},
$$

where $c$ is the speed of light, $k$ is the Boltzman constant, and $\lambda$ is the wavelength of the radiation.

The integral equation was solved numerically by dividing the medium into 1000 layers of unequal optical depths and assuming that the depth-dependent properties do not change within any one layer. The layering structure is a numerical technique only and is not intended to relate to any physical properties of the medium. The layer thickness increases from 0.001 optical depth per layer for the top 200 layers to $0.002,0.005,0.01$, and 0.02 , respectively, for increments of 200 layers. This procedure permits more accurate computation near the surface, where a large amount of the outgoing radiation originates. The calculations were not extended to the bottom of the ice sheet, but rather to 7.5 optical depths (corresponding to an average depth of $25 \mathrm{~m}$ ) because contributions to the outgoing radiation from further down were negligible.

Angular dependence of the intensity is controlled by the phase function, which, in this model, is the Rayleigh phase function given by:

$$
P(\theta)=(3 / 4)\left(1+\cos ^{2} \theta\right) \text {, }
$$

where $\theta$ is the angle between the scattered radiation and the direction of incidence. The angular variable was divided into 72 equal divisions, each of which is associated with an intensity that, in turn, is updated from layer to layer to provide the angular distribution of the radiant energy.

The numerical procedure is similar to the GaussSeidel iterative technique employed by Herman and Browning (1965) in atmospheric studies. Assuming isotropic up-welling radiation at the bottom of the medium, the calculation proceeded upward layer by layer. At each layer, the contributions of the upwelling components for each angle were summed, and the down-welling components were stored for a subsequent iteration. At the top layer, it was assumed that no downward radiation is received from outside the medium. The down-welling radiation was then calculated layer by layer, similar to the previous calculation but with sumnation of down-welling components and storage of up-welling values. This procedure was repeated until the percentage increment in net radiation at the surface was less than $0.05 \%$. The average number of $i$ terations that was made before convergence was about 10 . Various tests were made to check the accuracy of the numerical solution. One such test case is the calculation of emission as a function of angle from a pure scattering medium with Rayleigh phase function. The results from this model agreed very well with the analytic calculation of

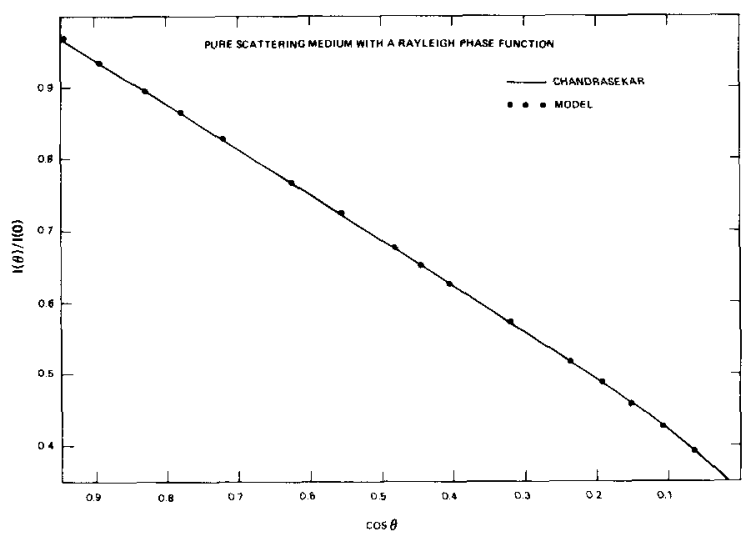

Fig.1. Comparison of model calculations with the analytic results of Chandrasekar (1960) for a pure scattering medium with a Rayleigh phase function.
Chandrasekhar (1960) as shown in Figure 1. Although the model calculates brightness temperature as a function of emission angle, the $T_{B}$ discussed below are nadir values to match the electrically scanning microwave radiometer (ESMR) observations.

\section{DEPTH-DEPENDENT PARAMETERS}

To evaluate the radiative transfer equation, the emittance, the single scattering albedo, and the physical temperature of the medium in each layer must be known. The emittance and the albedo at a particular optical depth $\tau$ are functions of the absorption coefficient $\gamma_{a}$ and the scattering coefficient $\gamma_{s}$ of the particle as follows:

$$
\begin{aligned}
& \varepsilon(\tau)=\frac{\gamma_{a}(\tau)}{\gamma_{e}(\tau)} \\
& \omega_{0}(\tau)=\frac{\gamma_{S}(\tau)}{\gamma_{e}(\tau)},
\end{aligned}
$$

where $\gamma_{e}=\gamma_{a}+\gamma_{s}$ is the extinction coefficient. The optical depth is given by $\tau(z)=\int_{0}^{z} \gamma_{e}(z) d z$, where $z$ is the depth in meters. For ice particles at a particular temperature, Mie scattering calculations show that $\gamma_{a}$ depends greatly on the imaginary part of the refractive index, $n^{\prime \prime}$, and is insensitive to crystal size for radi $i \leqslant 1 \mathrm{~mm}$ (Zwally 1977). Also, al though Cumming's (1952) measurements of the loss tangent at $3.2 \mathrm{~cm}$ indicate a strong dependence of the loss tangent on temperature from about 258 to about $273 \mathrm{~K}$, the $105 \mathrm{~s}$ tangent approaches an almost constant value at temperatures less than $258 \mathrm{~K}$. Because the temperatures relevant to this study are mostly less than $258 \mathrm{~K}$, the same value of $\gamma_{a}$ was therefore used at all seven stations and was kept constant as a function of depth in most of the calculations presented.

Nevertheless, both the magnitude of $n^{\prime \prime}$ and its temperature dependence at microwave wavelengths are not well known. A compilation by Evans (1965) shows some variations of the loss tangent with temperature down to about $213 \mathrm{~K}$. However, the measurements from different investigators in the compilation were not consistent with each other. The Debye equation (Hobbs

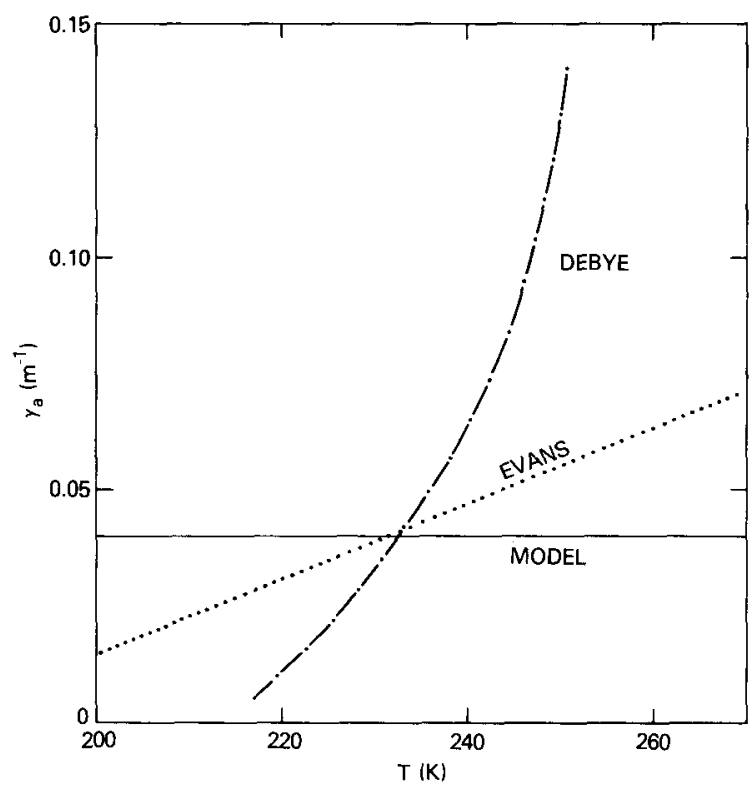

Fig.2. Temperature dependence of the coefficient of absorption as derived from the Debye equation and Evans (1965) at $1.55 \mathrm{~cm}$. A constant value was used in the model. 
1974:81-199) also shows a temperature dependence of the dielectric constant of ice, but, near the ESMR wavelength, it does not agree with Evans. Figure 2 compares interpolated $\gamma_{a}$ at $1.55 \mathrm{~cm}$ wavelength based on Evans' compilation, those derived from the Debye equation using an activation energy of $0.45 \mathrm{eV}$, and the constant value used in our model calculations. The absorption coefficient is considerably more sensitive to temperature in the Debye equation than in Evans' compilation. The Debye equation could also give a wide range of values for a particular temperature, depending on the activation energy, for which measured values for firn and ice range from 0.45 to $0.62 \mathrm{eV}$ (Hobbs 1974: 81-99). The effect of a possible temperature-dependent $\gamma_{a}$ on model results is discussed below.

The scattering coefficient $\gamma_{s}$ was determined from the size of the particles and the dielectric constant of the medium, assuming Rayleigh scattering (Van de Hulst 1957: 63-84). In this model, following Zwally (1977), we used the depth-dependent expression of $\gamma_{s}=(1.8 r)^{3}$ with $r^{3}=r_{0}+$ az based on Rayleigh scattering cross-sections and on the crystal size data of Gow (1969) for different snow depths and for various locations in Greenland and Antarctica.

To simulate the seasonal dependence of brightness temperature, a time-dependent temperature profile appropriate for the firn at Maudheim (Dalrymple and others 1966) was used:

$T(z, t)=T_{m}-A \exp [-0.3 z] \cos [0.99(t-84)-(97+20 z)]$,

where $T_{m}$ is the mean annual surface temperature, $t$ is the time, and (2A) is the amplitude at the surface. Although the temperature profile varies from one station to another, Equation (6) was used with appropriate values of $T_{m}$ and $A$ for each station.

\section{DISCUSSION OF RESULTS}

The model was tested for seven different locations in Greenland and Antarctica where depthdependent data are available. To compare results with the data, the mean bulk emissivity $\varepsilon=T_{B} / T_{m}$ (Zwally 1977) was determined for both observed and calculated brightness temperatures. The $T_{B}$ is a yearly average. The observed emissivity $\varepsilon$ (obs) was computed by using measured $\mathrm{T}_{\mathrm{m}}$ and an average of Nimbus-5 ESMR brightness temperature data from June 1973 to May 1975. Table I 1 ists the observed $\varepsilon$ (obs) values, which differ slightly from those reported by Zwally (1977) because they were determined from an improved and larger data set of observed brightness temperatures.
For $\varepsilon$ (model), $T_{m}$ was also used and $T$ was obtained from a solution of Equation (1). In a test case, $T_{B}$ was calculated for 12 months, using Equation (6) for the temperature profile. The average of the 12 monthly values was the same as that of the $T_{B}$ obtained by using a constant $T_{m}$ in Equation $(6)$ rather than a time-dependent profile. Therefore, to calculate the mean emissivity, it was not necessary to use a temperature profile, except for the case in which $\mathrm{Ya}_{\mathrm{a}}$ was made temperature-dependent. Density is another depth dependent parameter that was set at a constant value $480 \mathrm{~kg} \mathrm{~m}^{-3}$, as was done by Zwally (1977). In a test case, the firn density was varied $\pm 20 \%$, and the calculated variation in brightness temperature, and therefore emissivity, was $<2 \%$. This insensitivity to density occurs because both $\gamma_{a}$ and $\gamma_{s}$ are proportional to density so that their ratio is independent of density. Therefore, the variations in density from station to station and the variations in density with depth were neglected in the calculations of $T_{B}$.

The single most important variable in this model that affects the mean emissivities is the grain-size distribution that was used to calculate $\gamma_{s}$ at each location. Of the firn parameters considered, grain size is the parameter that varies most with location. The initial calculations using $\gamma_{a}=0.15 \mathrm{~m}^{-1}$ (based on Cumming's (1952) value of $\left.n^{\prime \prime}\right)^{a}$ and $\gamma_{s}=(1.8 r)^{3}$ gave calculated emissivities $\varepsilon$ (model) that differed significantly from $\varepsilon$ (obs) for most stations. Because there is substantial uncertainty in both $Y_{a}$ and $Y_{S}$, these coefficients were adjusted as follows. First, a value was chosen for the absorption coefficient $Y_{a}$ and an empirical factor $f$ was used to vary the scattering coefficient $\left(\gamma_{s}^{\prime}=f \gamma_{s}\right)$ until the differences between $\varepsilon$ (model) and $\varepsilon$ lobs) at four selected stations (South Pole, Plateau, South Ice, and Site 2) were minimized. The $\gamma_{s}$ was then kept constant, and $Y_{a}$ was varied until a minimum rms deviation was obtained. Figure 3 shows the effect of varying $r$. and $f$ at all stations on the calculated emissivities. Varying $\gamma_{a}$ with constant $\gamma_{s}$ changes the slope and the magnitude of the $\varepsilon$ (model) versus $\varepsilon$ (obs) relationship, whereas varying $\gamma_{s}$ with constant $\gamma_{a}$ primarily changes the magnitude of the $\varepsilon$ (model). At larger values of $\gamma_{a}$, the required value of $f$ increases but, contrary to the observations, the $\varepsilon$ (model) tends to be the same for all locations. This tendency occurs at larger values of $\gamma_{a}$ because the calculated radiation comes from nearer the surface, causing the sensitivity to variations in grain sizes at lower depths to decrease.

The values chosen for $f$ and $\gamma_{a}$ are 0.30 and

TABLE I. COMPARISON OF OBSERVED AND CALCULATED EMISSIVITIES

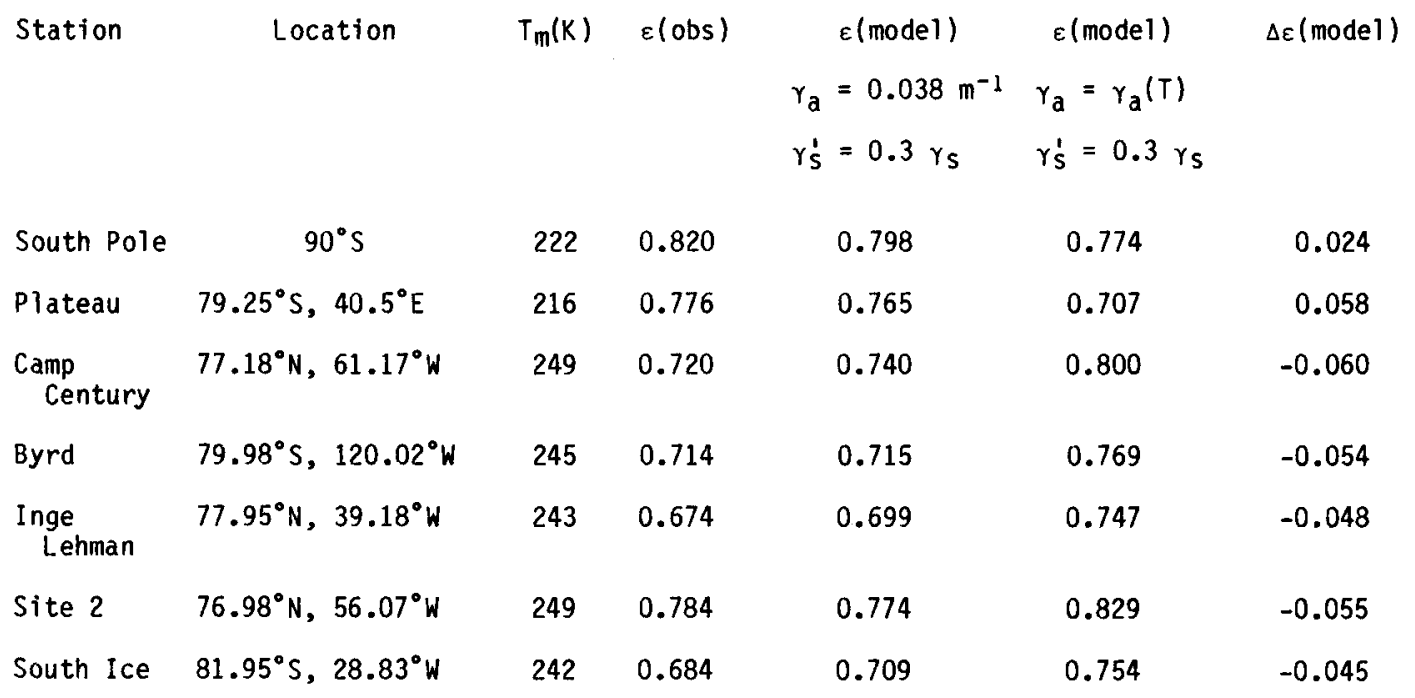




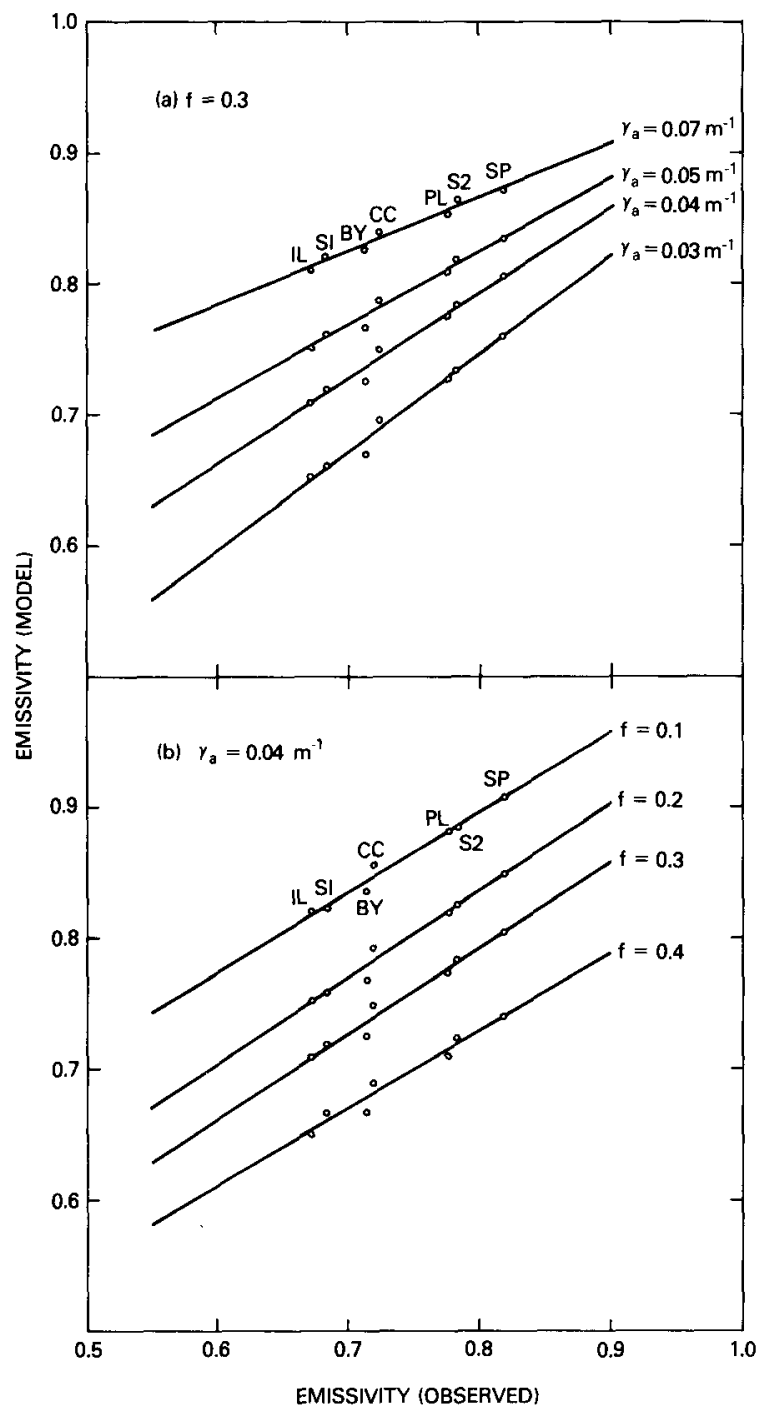

Fig.3. Observed versus modeled emissivities for various values of (a) absorption coefficients $\gamma_{a}$ and (b) scattering coefficients $\gamma_{a}^{\prime}=f \gamma_{s}$.

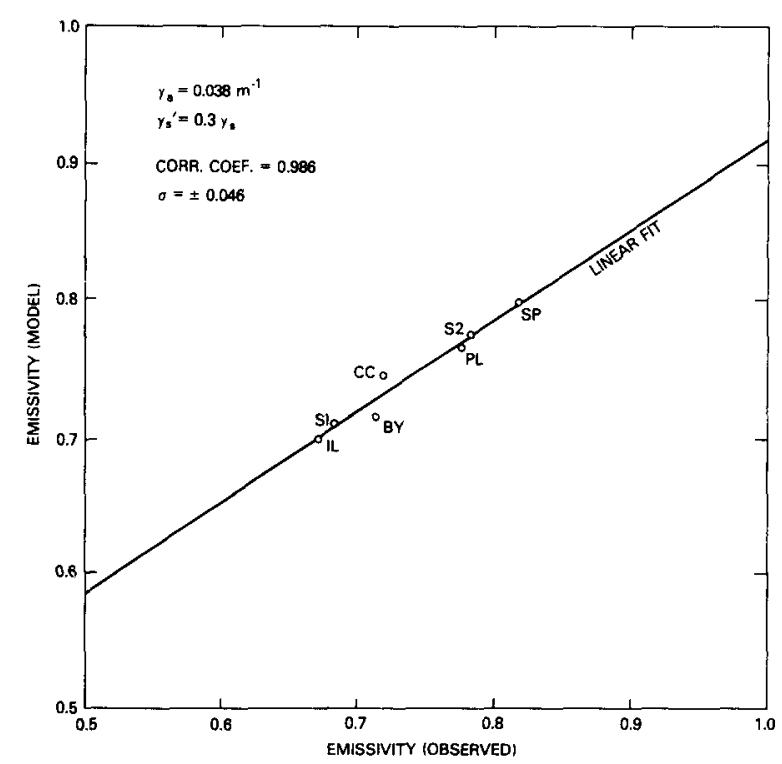

Fig.4. Comparison of observed and calculated mean emissivities at seven locations.
$0.038 \mathrm{~m}^{-1}$, and Figure 4 shows the resulting

correlation. The correlation coefficient is 0.986 , and the standard deviation is $4.6 \%$. This value of $f$ is roughly three times greater than that of Zwally's analytic solution, which required a small $f$ because of its overestimate of the effect of scattering. However, an $f$ less than unity means that the amount of scattering is still over-estimated in the model, despite the fact that multiple scattering was considered. Therefore, the calculated $\gamma_{5}$ apparently does not reflect the true scattering cross-sections of the snow grains in the firn. This suggests a limitation in the Rayleigh and Mie scattering models, which use a plane-wave approximation and assume independent spherical-scattering centers. Al though the absorption coefficient is a factor of four lower than the estimate made from the $3.2 \mathrm{~cm}$ data of Cumming (1952), it agrees well with the interpolated value from Evans (1965) at $1.55 \mathrm{~cm}$ wavelength and $232 \mathrm{~K}$.

As a further check on the reliability of the model and the choice of $\gamma_{a}$ and $\gamma_{s}$, the seasonal distribution of the brightness temperature was modeled. The results for Plateau and South Ice stations are shown in Figure 5. Plotted with the model results are the ESMR seasonal brightness temperature data for 1973, 1974, 1975, and 1976. These seasonal variations are useful because a large difference occurs in the depth-dependent temperature profile from summer to winter.

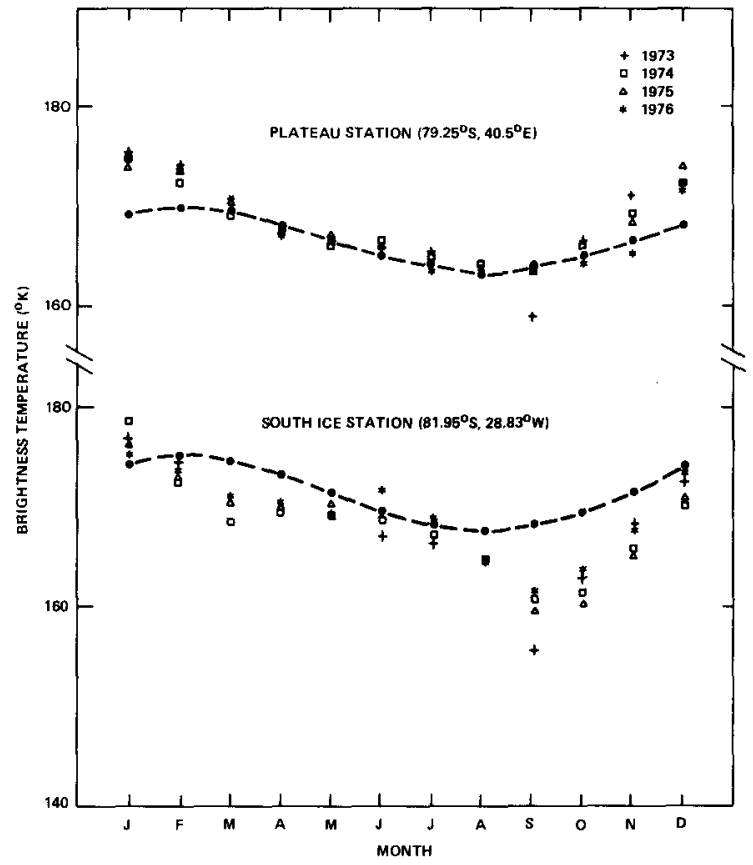

Fig.5. Observed and modeled seasonal variation of brightness temperatures at Plateau and South Ice stations. Model (dotted line) is compared with 4 a of ESMR $T_{B}$ data.

Because the amount of volume scattering controls the transmission of radiation from within the medium, the seasonal variation is an independent check on the $\gamma_{a}$ and $\gamma_{s}$ used in the model. For example, higher $\gamma_{a}$ gives higher seasonal amplitude because the radiation in that case comes from nearer the surface, where substantial seasonal variability occurs in the firn temperature. Except for September, October, and November, the agreement between theory and observed seasonal distribution for South Ice is good. The agreement is also good for Plateau except January, February, and December for which the data showed significantly higher values than the model. A better agreement could probably be obtained for Plateau by using a time-dependent temperature function approp- 
riate to the East Antarctic plateau. On the other hand, increasing the $\gamma$ to $0.15 \mathrm{~m}^{-1}$ for example, or using the Debye formula substantially increased the amplitude of the seasonal cycle above observed values. A better correlation for the emissivities in Figure 4 could be obtained by using a smaller absorption coefficient $\gamma_{a}=0.02 \mathrm{~m}^{-1}$ with $\gamma_{s}^{\prime}=0.12 \gamma_{s}$. However, this set of values would have a small seasonal variation in brightness temperature, and the correlation with observed data would not be as good. Furthermore, $\gamma_{a}=0.02 \mathrm{~m}^{-1}$ is consistently lower than both measurements from Cumming (1952) and Evans (1965), and $\gamma_{S}^{\prime}=0.12 \gamma_{S}$ is even further from calculated values.

A set of calculations was made using a temperature-dependent $\gamma_{a}$ that varied with station temperature. The temperature dependence of $\gamma_{a}$ in the Debye equation is quite strong, and using it in the model gave results that were inconsistent with observed emissivities and seasonal amplitudes of brightness temperature. The interpolation of dielectric constant from Evans (1965), shown in Figure 2, was also used to obtain a value of $\gamma_{a}$ based on the mean annual temperature for each of the seven stations. These values for $\gamma$ were also used in the model, and the enissivities obtained are shown in Table I. The results show that a good correlation at all seven stations is not possible with this temperaturedependent $\gamma_{a}$. This fact suggests that the temperature dependence of $\gamma_{a}$ is negligible as was assumed.

\section{CONCLUSION}

These results show that both the abnormally low brightness temperatures and the lack of simple correlation of these temperatures with physical temperatures can be successfully modeled by using a numerical solution of the radiative transfer equation with Rayleigh scattering. A consistently good correlation between the calculated and observed bulk emissivities at seven different stations can be obtained if the absorption coefficient, $\gamma_{a}$ is assumed to be identical at all these stations. This indicates that in the temperature range applicable for the ice sheet the temperature dependence of $r_{a}$ is either very small or negligible, which is consistent with the loss tangent measurements of Cumming (1952). The results also show that modeling of the seasonal dependence of brightness temperature can be a useful aid in selecting values of the scattering and absorption coefficients. In particular, the seasonal variation of brightness temperature is strongly dependent on emission depth, which, in turn, is controlled by the values of the scattering and absorption coefficients. Although the value chosen for $\gamma_{a}$ is quite consistent with some measurements of the index of refraction, the value for $\gamma_{s}$ obtained is substantially smaller $(\sim 70 \%)$ than Rayleigh scattering calculations based on measured grain size. This is not unexpected because the use of Rayleigh and Mie scattering models have some known limitations when applied to snow grains as scatterers. In particular, because the Rayleigh/Mie models use plane-wave approximations and assume independent spherical-scattering centers, exact quantitative agreement should not be expected.

\section{ACKNOWLEDGEMENT}

This research was supported by the National Aeronautics and Space Administration (NASA) Climate Prograin.

\section{REFERENCES}

Chandrasekhar S 1960 Radiative transfer. New York, Dover Publications Inc

Chang A $T C$, Gloersen $P$, Schmugge $T$, Wilheit $T T$, Zwally H J 1976 Microwave emission from snow and glacier ice. Journal of Glaciology 16(74): $23-39$
Chang A T C, Choudhury B J, Gloersen P 1980 Microwave brightness of polar firn as measured by Nimbus 5 and 6 ESMR. Journal of Glaciology 25(91): 85-91

Cumming $W A 1952$ The dielectric properties of ice and snow at 3.2 centimeters. Journal of Applied Physics 23(7): 768-773

Dalrymple $\mathrm{P} \mathrm{C}$, Lettau H H, Wollaston S H 1966 South Pole micrometeorology program: data analysis. In Rubin M J (ed) Studies in Antarctic meteorology. Washington, DC, American Geophysical Union: 13-57 (Antarctic Research Series 9)

Evans S 1965 Dielectric properties of ice and snow - a review. Joumal of Glaciology 5(42): 773-792

Herman B M, Browning S R 1965 A numerical solution to the equation of radiative transfer. Joumal of Atmospheric Sciences 22(5): 559-566

Gow A J 1969 on the rates of growth of grains and crystals in south polar firn. Joumal of Glaciology 8(53): 241-252

Hobbs P V 1974 Iee physics. Oxford, Clarendon Press

Swihart T L 1971 Basic physics of stezzar atmosphere. Tucson, Pachart Publishing House

Van de Hulst HC 1957 Light scattering from smazz particles. New York etc, John Wiley

Zwally H J 1977 Microwave emissivity and accumulation rate of polar firn. Joumal of Glaciology 18(79): 195-215

Zwally H J, Gloersen P 1977 Passive microwave images of the polar regions and research applications. Polar Record 18(116): 431-450 